\title{
WEIGHTED INEQUALITIES FOR RIEMANN-LIOUVILLE FRACTIONAL INTEGRALS OF ORDER ONE AND GREATER
}

\author{
F. J. MARTIN-REYES AND E. SAWYER
}

(Communicated by J. Marshall Ash)

\begin{abstract}
A simple characterization is given for two-weight norm inequalities for generalized Hardy operators $T_{\varphi} f(x)=\int_{0}^{x} \varphi\left(\frac{t}{x}\right) f(t) d t$, where $\varphi:(0,1) \rightarrow$ $(0, \infty)$ is nonincreasing and satisfies $\varphi(a b) \leq D[\varphi(a)+\varphi(b)]$ for $0<a, b<1$. Included in particular are the Riemann-Liouville fractional integrals.
\end{abstract}

\section{INTRODUCTION}

Let $T$ denote the Hardy operator, $T f(x)=\int_{0}^{x} f(t) d t$. If $w(x), v(x)$ are nonnegative functions on $(0, \infty)$ and if $1<p<\infty$, then (see M. Artola [A], B. Muckenhoupt [M], G. Talenti [Ta] and G. Tomaselli [To]) the weighted inequality,

$$
\int_{0}^{\infty}|T f(x)|^{p} w(x) d x \leq C \int_{0}^{\infty}|f(x)|^{p} v(x) d x, \text { for all } f,
$$

holds if and only if

$$
\sup _{r>0}\left(\int_{r}^{\infty} w(x) d x\right)^{1 / p}\left(\int_{0}^{r} v(x)^{1-p^{\prime}} d x\right)^{1 / p^{\prime}} \equiv A<\infty .
$$

Moreover, if $C$ is the least constant for which (1.1) holds, then $A \leq C \leq$ $p^{1 / p}\left(p^{\prime}\right)^{1 / p^{\prime}} A$.

In this note, we consider the weighted inequality (1.1) with the Hardy operator $T$ replaced by higher order antiderivatives, namely the Riemann-Liouville fractional integral operators,

$$
T_{\alpha} f(x)=\frac{1}{\Gamma(\alpha)} \int_{0}^{x}(x-t)^{\alpha-1} f(t) d t,
$$

for $\alpha \geq 1$. Note that for $\alpha \in \mathbf{N}, T_{\alpha}=T \circ T \circ \cdots T$, the Hardy operator iterated $\alpha$ times. Our result is that for $w(x), v(x) \geq 0$ on $(0, \infty)$ and $1<p<\infty$,

$$
\int_{0}^{\infty}\left|T_{\alpha} f(x)\right|^{p} w(x) d x \leq C \int_{0}^{\infty}|f(x)|^{p} v(x) d x, \text { for all } f,
$$

Received by the editors August 1, 1988 and, in revised from, October 25, 1988.

1980 Mathematics Subject Classification (1985 Revision). Primary 42B25.

The research of the first author was supported in part by C.A.I.C.Y.T. grant PB85-0434.

The research of the second author was supported in part by NSERC grant A5149. 
if and only if both of the following conditions hold (1.4)

$$
\sup _{r>0}\left(\int_{r}^{\infty} w(x)\left(\frac{x}{r}\right)^{p(\alpha-1)} d x\right)^{1 / p}\left(\int_{0}^{r}(r-t)^{p^{\prime}(\alpha-1)} v(t)^{1-p^{\prime}} d t\right)^{1 / p^{\prime}} \equiv A<\infty,
$$

$$
\sup _{r>0}\left(\int_{r}^{\infty} w(x)(x-r)^{p(\alpha-1)} d x\right)^{1 / p}\left(\int_{0}^{r} v(t)^{1-p^{\prime}} d t\right)^{1 / p^{\prime}} \equiv B<\infty .
$$

In the case $\alpha=1,(1.4)$ and (1.5) both reduce to (1.2), but if $\alpha>1$, then neither (1.4) nor (1.5) alone is in general sufficient for (1.3)-counterexamples are given below.

We actually prove a more general theorem. Let

$$
T_{\varphi}(f \sigma)(x)=\int_{0}^{x} \varphi\left(\frac{t}{x}\right) f(t) d \sigma(t), \quad x>0,
$$

where $\varphi:(0,1) \rightarrow(0, \infty), f(t) \geq 0$ and $\sigma$ is a positive Borel measure on $(0, \infty)$. Here and throughout this paper, $\int_{a}^{b} g d \sigma$ is to be interpreted as $\int_{[a, b]} g d \sigma$ whenever $\sigma$ has an atomic component.

Theorem. Suppose $1<p \leq q<\infty$ and that $\omega$ and $\sigma$ are locally finite positive Borel measures on $(0, \infty)$. Suppose further that $\varphi:(0,1) \rightarrow(0, \infty)$ is nonincreasing and satisfies

$$
\varphi(a b) \leq D[\varphi(a)+\varphi(b)], \quad \text { for } 0<a, b<1 .
$$

Then

$$
\left(\int_{0}^{\infty}\left[T_{\varphi}(f \sigma)(x)\right]^{q} d \omega(x)\right)^{1 / q} \leq C\left(\int_{0}^{\infty} f(x)^{p} d \sigma(x)\right)^{1 / p}, \text { for all } f \geq 0,
$$

if and only if both of the following conditions hold

$$
\begin{aligned}
& \sup _{r>0}\left(\int_{r}^{\infty} d \omega\right)^{1 / q}\left(\int_{0}^{r} \varphi\left(\frac{t}{r}\right)^{p^{\prime}} d \sigma(t)\right)^{1 / p^{\prime}} \equiv A<\infty, \\
& \sup _{r>0}\left(\int_{r}^{\infty} \varphi\left(\frac{r}{x}\right)^{q} d \omega(x)\right)^{1 / q}\left(\int_{0}^{r} d \sigma\right)^{1 / p^{\prime}} \equiv B<\infty .
\end{aligned}
$$

Moreover, if $C$ is the least constant for which (1.7) holds, then the ratio $C /(A+B)$ is bounded between two positive constants depending only on $p$ and the constant $D$ in (1.6).

The case $\varphi \equiv 1$ of this theorem is in J. Bradley [B] and K. Andersen and B. Muckenhoupt [AM]. The theorem includes the result for the RiemannLiouville fractional integrals mentioned earlier. To see this, take $\varphi(x)=$ $(1-s)^{\alpha-1} / \Gamma(\alpha)$ (then (1.6) holds with $D=\max \left\{1,2^{\alpha-2}\right\}$ since $1-x y \leq 1-x y+$ $(1-x)(1-y)=(1-x)+(1-y)$ for $0 \leq x, y \leq 1), p=q, d \omega(x)=$ $w(x) x^{p(\alpha-1)} d x$ and $d \sigma(t)=v(t)^{1-p^{\prime}} d t$ in the theorem and then replace $f(t)$ 
by $f(t) v(t)^{p^{\prime}-1}$ in (1.7) to get (1.3). The proof of the theorem is in $\S 2$ and some examples are given in $\S 3$ to show that in general, neither (1.8) nor (1.9) alone is sufficient for the weighted inequality (1.7). In fact, (1.8) and (1.9) are equivalent if and only if $\varphi$ is bounded from above and below by positive constants.

\section{Proof of THE THeOREM}

Suppose first that $(1.7)$ holds and that $\varphi:(0,1) \rightarrow(0, \infty)$ is nonincreasing. We show that both (1.8) and (1.9) follow. To see (1.8), fix $r>0$ and let $f(t)=\chi_{(0, r]}(t) \varphi(t / r)^{p^{\prime}-1}$. Then for $x \geq r$, we have $\varphi(t / r) \leq \varphi(t / x)$ and so

$$
\begin{aligned}
& \left(\int_{r}^{\infty} d \omega\right)\left(\int_{0}^{r} \varphi\left(\frac{t}{r}\right)^{p^{\prime}} d \sigma(t)\right)^{q} \\
& \quad \leq \int_{r}^{\infty}\left(\int_{0}^{r} \varphi\left(\frac{t}{x}\right) \varphi\left(\frac{t}{r}\right)^{p^{\prime}-1} d \sigma(t)\right)^{q} d \omega(x) \\
& \quad \leq \int_{r}^{\infty}\left(T_{\varphi}(f \sigma)(x)\right)^{q} d \omega(x) \\
& \quad \leq C^{q}\left(\int_{0}^{\infty} f^{p} d \sigma\right)^{q / p}=C^{q}\left(\int_{0}^{r} \varphi\left(\frac{t}{r}\right)^{p^{\prime}} d \sigma(t)\right)^{q / p} .
\end{aligned}
$$

Now divide through by the right hand side and take $q$ th roots to obtain (1.8)if the right-hand side is 0 or $\infty$, then the arguments of [M] show that (1.8) is trivially true with the convention $0 \cdot \infty=0$. To obtain (1.9) we consider the equivalent inequality dual to (1.7):

$$
\left(\int_{0}^{\infty}\left[T_{\varphi}^{*}(g \omega)(x)\right]^{p^{\prime}} d \sigma(x)\right)^{1 / p^{\prime}} \leq C\left(\int_{0}^{\infty} g(x)^{q^{\prime}} d \omega(x)\right)^{1 / q^{\prime}}, \text { for all } g \geq 0 \text {, }
$$

where $T_{\varphi}^{*}(g \omega)(x)=\int_{x}^{\infty} \varphi(x / t) g(t) d \omega(t)$. Setting $g(t)=\chi_{[r, \infty)}(t) \varphi(r / t)^{q-1}$ in (2.1) and arguing as above, we obtain (1.9).

Conversely, suppose (1.8) and (1.9) hold and that $\varphi$ is nonincreasing and satisfies (1.6). Let $f(t)$ be nonnegative, bounded and compactly supported in $(0, \infty)$-if we prove (1.7) for such $f$ with a constant $C$ independent of $f$, then the monotone convergence theorem will complete the proof. Now $T_{\varphi}(f \sigma)$ is nondecreasing on $(0, \infty)$, and finite on $(0, a)$ whenever $\int_{a}^{\infty} d \omega>0$ by Hölder's inequality and condition (1.8). With $D$ as in (1.6), choose $x_{k}$ for $k$ as large as possible, say $-\infty<k<N, N \in \mathbf{N} \cup\{\infty\}$, to satisfy

$$
T_{\varphi}(f \sigma)\left(x_{k}\right)=\int_{0}^{x_{k}} \varphi\left(\frac{t}{x_{k}}\right) f(t) d \sigma(t)=(D+1)^{k}, \quad k \in \mathbf{Z} .
$$

(Routine but tedious modifications, which we omit, are needed if $\sigma$ has an atomic component.) Then $\left\{x_{k}\right\}_{-\infty<k<N}$ is an increasing sequence of positive 
numbers and

$$
\begin{aligned}
&(D+1)^{k-1}=(D+1)^{k}-D(D+1)^{k-1} \\
& \quad=\int_{0}^{x_{k}} \varphi\left(\frac{t}{x_{k}}\right) f(t) d \sigma(t)-D \int_{0}^{x_{k-1}} \varphi\left(\frac{t}{x_{k-1}}\right) f(t) d \sigma(t) \\
& \quad=\int_{0}^{x_{k-1}}\left(\varphi\left(\frac{t}{x_{k}}\right)-D \varphi\left(\frac{t}{x_{k-1}}\right)\right) f(t) d \sigma(t)+\int_{x_{k-1}}^{x_{k}} \varphi\left(\frac{t}{x_{k}}\right) f(t) d \sigma(t) \\
& \leq D \int_{0}^{x_{k-1}} \varphi\left(\frac{x_{k-1}}{x_{k}}\right) f(t) d \sigma(t)+\int_{x_{k-1}}^{x_{k}} \varphi\left(\frac{t}{x_{k}}\right) f(t) d \sigma(t)
\end{aligned}
$$

by (1.6) with $a=t / x_{k-1}$ and $b=x_{k-1} / x_{k}$. Note for later use that in the case $\varphi \equiv 1$, the first summand on the right side above may be dropped. Now suppose $N=\infty$; the case $N<\infty$ requires an easy modification of the following argument. Then

$$
\begin{aligned}
\int_{0}^{\infty}[ & \left.T_{\varphi}(f \sigma)(x)\right]^{q} d \omega(x) \leq \sum_{k} \int_{x_{k}}^{x_{k+1}}(D+1)^{(k+1) q} d \omega \\
\leq & C_{q} D^{3 q} \sum_{k}\left(\int_{x_{k}}^{x_{k+1}} d \omega\right)\left(\int_{0}^{x_{k-1}} \varphi\left(\frac{x_{k-1}}{x_{k}}\right) f(t) d \sigma(t)\right)^{q} \\
& +C_{q} D^{2 q} \sum_{k}\left(\int_{x_{k}}^{x_{k+1}} d \omega\right)\left(\int_{x_{k-1}}^{x_{k}} \varphi\left(\frac{t}{x_{k}}\right) f(t) d \sigma(t)\right)^{q} \\
= & \text { I }+ \text { II. }
\end{aligned}
$$

Now

$$
\begin{aligned}
\mathrm{II} \leq C_{q} D^{2 q} \sum_{k}\left(\int_{x_{k}}^{x_{k+1}} d \omega\right)\left(\int_{x_{k-1}}^{x_{k}} \varphi\left(\frac{t}{x_{k}}\right)^{p^{\prime}} d \sigma(t)\right)^{q / p^{\prime}} \\
\quad \times\left(\int_{x_{k-1}}^{x_{k}} f(t)^{p} d \sigma(t)\right)^{q / p}
\end{aligned}
$$

by Hölders inequality,

$$
\begin{aligned}
& \leq C_{q} D^{2 q} A^{q} \sum_{k}\left(\int_{x_{k-1}}^{x_{k}} f(t)^{p} d \sigma(t)\right)^{q / p}, \quad \text { by }(1.8), \\
& \leq C_{q} D^{2 q} A^{q}\left(\sum_{k} \int_{x_{k-1}}^{x_{k}} f(t)^{p} d \sigma(t)\right)^{q / p} \quad \text { since } p \leq q \\
& \leq C_{q} D^{2 q} A^{q}\left(\int_{0}^{\infty} f(t)^{p} d \sigma(t)\right)^{q / p} .
\end{aligned}
$$

At this point we observe that if $\varphi \equiv 1$, i.e. $T_{\varphi}$ is the Hardy operator, then term I is absent and the above estimate for II together with (2.2) yields (1.7). 
Thus we have shown,

$$
\begin{aligned}
& \left(\int_{0}^{\infty}\left(\int_{0}^{x} f d \sigma\right)^{q} d \omega(x)\right)^{1 / q} \\
& \quad \leq C_{p, q}\left\{\sup _{r>0}\left(\int_{r}^{\infty} d \omega\right)^{1 / q}\left(\int_{0}^{r} d \sigma\right)^{1 / p^{\prime}}\right\}\left(\int_{0}^{\infty} f^{p} d \sigma\right)^{1 / p}, \text { for all } f \geq 0,
\end{aligned}
$$

for $1<p \leq q<\infty$ and locally finite positive Borel measures $\omega$ and $\sigma$. We now return to the proof of the theorem and use (2.3) to estimate term I as follows. With $d \delta_{a}$ the point mass at $a$, we have

$$
\begin{aligned}
\mathrm{I} & =C_{q} D^{3 q} \sum_{k}\left(\int_{x_{k}}^{x_{k+1}} \varphi\left(\frac{x_{k-1}}{x_{k}}\right)^{q} d \omega(x)\right)\left(\int_{0}^{x_{k-1}} f d \sigma\right)^{q} \\
& =C_{q} D^{3 q} \int_{0}^{\infty}\left(\int_{0}^{y} f d \sigma\right)^{q} \sum_{k}\left(\int_{x_{k}}^{x_{k+1}} \varphi\left(\frac{x_{k-1}}{x_{k}}\right)^{q} d \omega(x)\right) d \delta_{x_{k-1}}(y) \\
& \leq C_{q} D^{3 q}\left(C_{p, q}\right)^{q} E^{q}\left(\int_{0}^{\infty} f^{p} d \sigma\right)^{q / p}
\end{aligned}
$$

where

$$
\begin{aligned}
E= & \sup _{r>0}\left[\int_{r}^{\infty} \sum_{k}\left(\int_{x_{k}}^{x_{k+1}} \varphi\left(\frac{x_{k-1}}{x_{k}}\right)^{q} d \omega(x)\right) d \delta_{x_{k-1}}\right]^{1 / q} \\
& \times\left(\int_{0}^{r} d \sigma\right)^{1 / p^{\prime}}, \quad \text { by }(2.3), \\
\leq & 2^{1 / q} \sup _{r>0}\left[\int_{r}^{\infty} \varphi\left(\frac{r}{x}\right)^{q} d \omega(x)\right]^{1 / q}\left(\int_{0}^{r} d \sigma\right)^{1 / p^{\prime}}=2^{1 / q} B, \quad \text { by }(1.9),
\end{aligned}
$$

since $\varphi\left(x_{k-1} / x_{k}\right) \leq \varphi(r / x)$ for $r \leq x_{k-1}<x_{k} \leq x$. Combining the estimates for I and II with (2.2) yields (1.7) and this completes the proof of the theorem.

\section{COUNTEREXAMPLES}

Let $d \sigma=d \delta_{1}$, the unit point mass at 1 . Then (1.8) becomes

$$
\left(\int_{r}^{\infty} d \omega\right)^{1 / q} \varphi\left(\frac{1}{r}\right) \chi_{[1, \infty)}(r) \leq A, \text { for all } r>0
$$

Solving for equality in (3.1) with $A=1$ we obtain $\int_{r}^{\infty} d \omega(x)=\varphi(1 / r)^{-q}$, $r \geq 1$, or

$$
\frac{d \omega}{d x}(r)=-q \frac{\varphi^{\prime}(1 / r)}{\varphi(1 / r)^{q+1}} \frac{1}{r^{2}} .
$$

Thus (1.8) holds with $A=1$ if $d \sigma=d \delta_{1}$ and

$$
d \omega(x)=-\chi_{[1, \infty)}(x) \frac{q \varphi^{\prime}(1 / x)}{\varphi(1 / x)^{q+1}} \frac{d x}{x^{2}}
$$

(even if $\varphi(0+)<\infty$-we tacitly assumed $\varphi(0+)=\infty$ in our derivation). 
However,

$$
\begin{aligned}
\int_{1}^{\infty} \varphi\left(\frac{1}{x}\right)^{q} d \omega(x) & =-q \int_{1}^{\infty} \varphi\left(\frac{1}{x}\right)^{q} \frac{\varphi^{\prime}(1 / x)}{\varphi(1 / x)^{q+1}} \frac{d x}{x^{2}} \\
& =\left.q \log \varphi\left(\frac{1}{x}\right)\right|_{1} ^{\infty}=q \log \frac{\varphi(0+)}{\varphi(1-)}
\end{aligned}
$$

and so (1.9) fails if $\varphi(0+) / \varphi(1-)=\infty$, i.e. if $\varphi$ fails to be bounded from above and below by positive constants. Similarly, (1.9) holds with $B=1$ if $d \omega=d \delta_{1}$ and $d \sigma(t)=-\chi_{(0,1]}(t)\left(p^{\prime} \varphi^{\prime}(t) / \varphi(t)^{p^{\prime}+1}\right) d t$, and (1.8) then fails if $\varphi(0+) / \varphi(1-)=\infty$. It follows that conditions (1.8) and (1.9) are equivalent if and only if $\varphi$ is bounded from above and below by positive constants, i.e. $T_{\varphi}$ is essentially the Hardy operator.

The Dirac measures in the above examples can be replaced by weights. For example, if $\varphi$ is decreasing and absolutely continuous on $(0,1)$ with $\varphi(0+)<$ $\infty$ and $\varphi(1-)=0$ (as is the case for the fractional integral $T_{\alpha}, \alpha>1$ ), let as above

$$
\begin{aligned}
d \omega(x) & =-\chi_{(1, \infty)}(x) d\left[\varphi\left(\frac{1}{x}\right)^{-q}\right] \\
& =-\chi_{(1, \infty)}(x) q \varphi\left(\frac{1}{x}\right)^{-q-1} \varphi^{\prime}\left(\frac{1}{x}\right) x^{-2} d x
\end{aligned}
$$

but replace $d \sigma=d \delta_{1}$ by

$$
d \sigma(t)=\chi_{(1, \infty)}(t) d\left[1+\log \left[\frac{\varphi(0+)}{\varphi\left(t^{-2}\right)}\right]\right]^{-\delta}
$$

where $0<\delta<p^{\prime} / q$. Then for $r>1$, we have

$$
\begin{gathered}
\int_{r}^{\infty} d \omega(x)=-\left.\varphi\left(\frac{1}{x}\right)^{-q}\right|_{r} ^{\infty}=\varphi\left(\frac{1}{r}\right)^{-q}-\varphi(0+)^{-q} \leq \varphi\left(\frac{1}{r}\right)^{-q} \\
\int_{0}^{r} d \sigma(t)=\left.\left[1+\log \left[\frac{\varphi(0+)}{\varphi\left(t^{-2}\right)}\right]\right]^{-\delta}\right|_{1} ^{r}=\left[1+\log \left[\frac{\varphi(0+)}{\varphi\left(r^{-2}\right)}\right]\right]^{-\delta}
\end{gathered}
$$

and

$$
\begin{aligned}
\int_{0}^{r} \varphi\left(\frac{t}{r}\right)^{p^{\prime}} d \sigma(t) & =\int_{1}^{r} \varphi\left(\frac{t}{r}\right)^{p^{\prime}} d \sigma(t) \leq \varphi\left(\frac{1}{r}\right)^{p^{\prime}} \int_{1}^{r} d \sigma(t) \\
& \leq \varphi\left(\frac{1}{r}\right)^{p^{\prime}} \int_{0}^{\infty} d \sigma(t)=\varphi\left(\frac{1}{r}\right)^{p^{\prime}} .
\end{aligned}
$$


Now for $x \geq r^{2} \geq 1$, we have $\varphi(1 / x) \leq \varphi\left(1 / r^{2}\right) \leq 2 D \varphi(1 / r) \leq 2 D \varphi(r / x)$ by (1.6) and since $\varphi$ is decreasing. Thus

$$
\begin{aligned}
\int_{r}^{\infty} \varphi\left(\frac{r}{x}\right)^{q} d \omega(x) & \geq(2 D)^{-q} \int_{r^{2}}^{\infty} \varphi\left(\frac{1}{x}\right)^{q} d \omega(x) \\
& =-q(2 D)^{-q} \int_{r^{2}}^{\infty} \varphi\left(\frac{1}{x}\right)^{-1} \varphi^{\prime}\left(\frac{1}{x}\right) x^{-2} d x \\
& =q(2 D)^{-q} \log \left[\frac{\varphi(0+)}{\varphi\left(r^{-2}\right)}\right],
\end{aligned}
$$

and so (1.8) holds, but not (1.9) since $\delta<p^{\prime} / q$. A similar construction shows there is a pair of weights such that (1.9) holds, but not (1.8). In particular, we see that for $\alpha>1$, neither (1.4) nor (1.5) alone is sufficient for (1.3).

Finally we remark that these examples can be further modified to produce weights that are positive and finite almost everywhere on $(0, \infty)$, and satisfy one of (1.8), (1.9) but not the other. The idea is to construct, for each $0<a<1$, analogues of the above weights, starting the construction with $d \delta_{a}$ in place of $d \delta_{1}$, and then to add up appropriate multiples of truncations of these weights for a sequence of $a$ 's tending to zero. The tedious details are omitted.

Added in proof. We wish to thank Professor V. Stepanov for sending us his paper [S] in which he showed that the Riemann-Liouville fractional integral operator satisfies (1.3) if and only if both (1.5) and

$$
(1.4)^{\prime} \sup _{r>0}\left(\int_{r}^{\infty} w(x) d x\right)^{1 / p}\left(\int_{0}^{r}(r-t)^{p^{\prime}(\alpha-1)} v(t)^{1-p^{\prime}} d t\right)^{1 / p^{\prime}} \equiv A^{\prime}<\infty
$$

hold. We also thank Professor J. Garcia-Cuerva for independently pointing out to us that condition (1.4) can be replaced by $(1.4)^{\prime}$ in this context.

\section{REFERENCES}

[AM] K. Andersen and B. Muckenhoupt, Weighted weak type Hardy inequalities with applications to Hilbert transforms and maximal functions, Studia Math. 72 (1982), 9-26.

[A] M. Artola, untitled and unpublished manuscript.

[B] J. S. Bradley, Hardy inequalities with mixed norms, Canad. Math. Bull. 21 (1978), 405-408.

[M] B. Muckenhoupt, Hardy's inequality with weights, Studia Math. 34 (1972), 31-38.

[S] V. Stepanov, Two-weighted estimates for Riemann-Liouville integrals, Ceskoslovenska Akademie Vid 39 (1988), 1-28.

[Ta] G. Talenti, Osservazioni sopra una classe di disuguaglianze, Rend. Sem. Mat. Fis. Milano 39 (1969), 171-185.

[To] G. Tomaselli, A class of inequalities, Boll. Un. Mat. Ital. 2 (1969), 622-631.

Departamento de Análisis Matemático, Facultad de Ciencias, Universidad de Málaga, 29071-Málaga, Spain

Department of Mathematics and Statistics, McMaster University, Hamilton, ONTARIO L85 4K1, CANADA 